\title{
Shielding Analysis of High Frequency Coaxial Transformers Used for Electric Vehicle On-Board Charging Systems
}

\author{
W.Water ${ }^{1}$, Student Member, IEEE, J. Lu ${ }^{1}$, Senior Member, IEEE \\ ${ }^{1}$ School of Engineering, Griffith University, Nathan, QLD 4111, Australia
}

\begin{abstract}
This paper introduces a High Frequency Coaxial Transformer (HFCT) with a power efficiency of $>99.5 \%$ and an operational frequency between $100-300 \mathrm{kHz}$. The power rating of the HFCT can be easily scaled from 2.5 to $20 \mathrm{~kW}$ by adapting its simple symmetrical structure. The introduced HFCT is suitable for use with converters in Electric Vehicle (EV) on-board charging systems. It easily fulfills the design requirements of being light-weight, compact, and having a high power density and achieved a volume reduction of $75 \%$ in comparison with commercial products. Furthermore, the electromagnetic interferences are increasing with the increased operating frequency, and an inserted copper Faraday shield is used to deal with this issue (an $80.6 \%$ reduction of intra-winding capacitance was achieved). A shielding analysis, with a focus on the insertion losses, is undertaken by utilizing Finite Element Method (FEM) based numerical techniques.
\end{abstract}

Index Terms - charging system, electromagnetic interference, electric vehicle, Faraday shield, finite element method, high frequency coaxial transformer, smart grid.

\section{INTRODUCTION}

$\mathrm{T}$ he Electric Vehicle (EV) occupies an important role in smart grid infrastructure (the integration of renewable energies, such as household and power grids), and captures interest because of its potential usage in the near future. Several researchers have discussed future trends and requirements of the EV [1-2]. Summarising this information can make one short conclusion: the quality of charging process is very important for publics to accept and get used to the EV technology. Several charging standards were developed such as SAE, IEC and ISO, which have varied definitions on port communication and power rating [3]. To make it simple, we can classify charging standards (according to its power rating) into three categories. These include normal charging, fast charging and super charging, with power ratings of $1-2 \mathrm{~kW}, 2-20 \mathrm{~kW}$ and $>20 \mathrm{~kW}$ respectively (approximation) [1-4]. A lot of research has been undertaken on normal charging levels at both low frequency (60 to $100 \mathrm{~Hz}$ ) and high frequency (1 to $10 \mathrm{kHz}$ and above). However, very little research has been studied for power

Manuscript received December 23, 2012. This work was supported in part by the Australia Research Council Discovery Project (ID: DP1094543). W. Water is with the Engineering of Griffith University, Nathan, QLD, AUS. (phone: +61-423-469504; e-mail: wayne_water@ hotmail.com). conversion applications in the EV above an operating frequency of $100 \mathrm{kHz}$ and power rating greater than $2 \mathrm{~kW}$. The power converter commonly seen in the EV market operates at $60 \mathrm{~Hz}$ to $200 \mathrm{~Hz}$, in which the converter is bulky and inefficient. Therefore, this paper introduces an isolated HFCT, which is well-suited for EV charging systems at fast charging level, as well as other energy storage applications. The benefit of using an isolated transformer in the converter system has been discussed in several papers [5-7]. On the other hand, the coupling capacitance accompanied by an increased operating frequency (which couples HF noise between the primary and secondary windings) can cause serious common mode problems [8-10]. Hence, a Faraday shield is placed between the windings of the HFCT. This reduces the coupling capacitance and consequently the electromagnetic interference (EMI). The analysis of the shielding effect of the HFCT is presented in this paper and maybe of interest to other researchers and automobile manufacturers.

\section{NUMERICAL MODELING}

\section{A. FEM Based Numerical Method}

A Finite Element Method (FEM) frequency domain based numerical technique was used to analyse the eddy current and magnetic flux distribution for the $\mathrm{HF}$ transformer. The transformer's nonlinear magnetic field can be determined by the following vector potential equation:

$$
\nabla \times(v \nabla \times A)+\sigma(\partial A / \partial t)=J
$$

where $A$ and $J$ are magnetic vector potential and current density respectively, $v$ is the magnetic reluctivity, and $\sigma$ is the conductivity. The energy function is generalised from linear techniques. The energy method has been chosen to solve the inductance of the HFCT. From (1), the corresponding magnetic field is obtained with the use of the FEM technique for the whole model. Therefore, energy stores in the HFCT are also obtained:

$$
W_{m}=\frac{1}{2} \int_{\Omega} B \cdot H d \Omega
$$

where in a $\mathrm{N}$-turn winding system, $B$ is the flux density, $H$ is the magnetic intensity and $\Omega$ is the area enclosed by the generated magnetic field. 
To solve the capacitance model of the HFCT, the energy method is still applicable. However, solving it with the charge equation is comparably easier and reduces the computation time. The capacitance computation is based on Maxwell's theory, and the relationship between potential and charge in a multi-conductor system can be described by the electric scalar potential $V$, which satisfies Poisson's equation:

$$
-\nabla \cdot(\varepsilon \nabla V)=\rho
$$

where $\varepsilon$ is the permittivity, and $\rho$ is the space charge density. By finding the number of charges in each individual conductor, the unknown capacitance can be obtained.

\section{B. HFCT Structure Configuration}

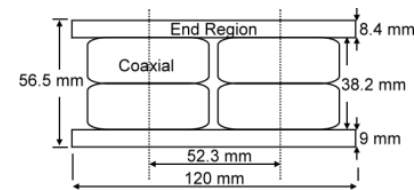

(a)

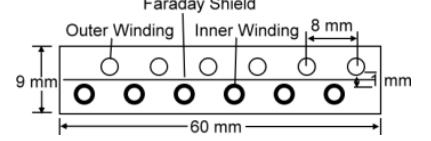

(b)

Fig.1. An 8 kW HFCT schematic: (a) Front view; (b) End region cross section; and (c) Top view of half cross-section on the coaxial part.

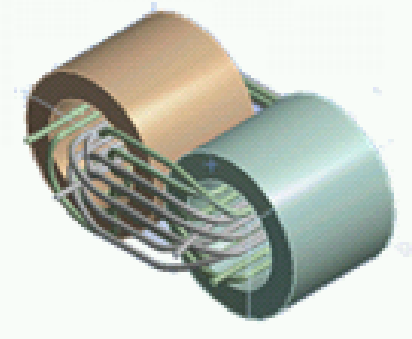

(a) Without shield

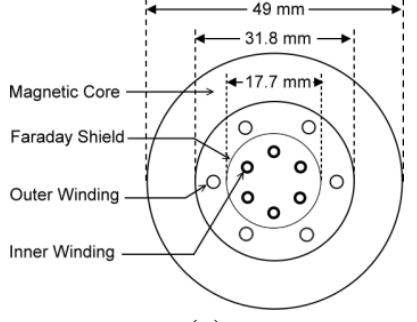

(c)

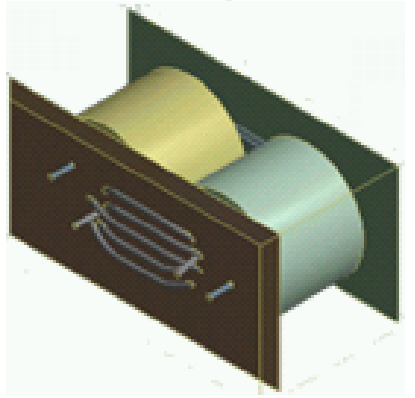

(b) With shield
Fig.2. The 3D HFCT simulation model with and without the Faraday shield.

An $8 \mathrm{~kW}$ HFCT prototype is used as the bench model throughout this paper and the detailed geometrical structure is shown in Fig. 1. Dimensions in this figure are only for an $8 \mathrm{~kW}$ HFCT prototype and can be scaled according to the required power rating. Both $2 \mathrm{D}$ and $3 \mathrm{D}$ simulations have been conducted and are presented in this paper, and the dimension is provided in Fig. 1. The thickness of the Faraday shield is 0.1 $\mathrm{mm}$. Please refer to Fig.1 (a) for the definitions of a coaxial region and an end region as they will be broadly used in the simulation section. Fig. 2 shows the 3D model of the HFCT with and without the Faraday shield insertion. There are four pieces of copper shield for the HFCT with the Faraday shield: two circular rings in the centre of the coaxial section and the other two in the end region section to isolate the inner and outer winding. The material of the magnetic ring is ferrite, which has a high initial relative permeability of 2100 and a maximum flux density of $0.4 \mathrm{~T}$.

\section{Testing Results}

The HFCT equivalent circuit is shown in Fig.3, which illustrates the transformer parameters used in testing and simulation results. The transformer parameters were obtained by using a HP $4285 \mathrm{~A}(75 \mathrm{kHz}$ to $30 \mathrm{MHz})$ precision LCR meter.
Testing results are tabulated in Table I and Table II where $\mathrm{L}_{\mathrm{s}}$ and $\mathrm{R}_{\mathrm{s}}$ denote total leakage inductance and total winding resistance, $L_{m}$ denotes magnetizing inductance and $R_{c}$ denotes core loss resistance respectively. (a) Without shield

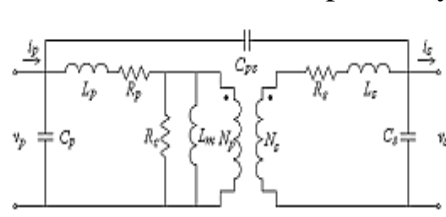

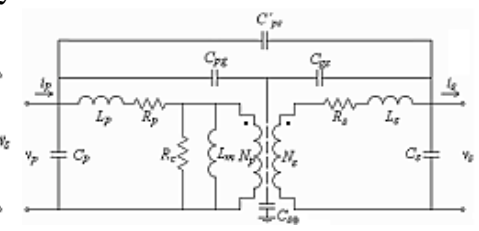

(b) With shield
Fig.3. Simplified HFCT equivalent circuit and parameters.

All parameters are frequency dependent, and the insertion of the Faraday shield has very little negative effect on the power efficiency performance. The leakage inductance increases slightly (approximately $7.2 \%$ ) due to the finite amount of space required for the insertion of the Faraday shield. However, the variation is too small to be significant. The $\mathrm{L}_{\mathrm{m}}$ has a minor decrease with the insertion of the Faraday shield while an increase is shown in $\mathrm{L}_{\mathrm{s}}$. This is reasonable since the material of the Faraday shield is copper (which counteracts the magnetic field and consequently the electric field). Also, energy distributes in the magnetic core or in the air, which represents $\mathrm{L}_{\mathrm{m}}$ and $\mathrm{L}_{\mathrm{s}}$ respectively. From an energy point of view, the magnetizing inductance and leakage inductance have an inversely proportional relationship to each other. Furthermore, the $R_{s}$ variation in Table II shows the eddy current effect in that the wire resistance increases with the frequency in an exponential relationship.

TABLE I

OPEN CIRCUIT TESTING RESUlTS OF THE COAXIAL TRANSFORMER

\begin{tabular}{rcccc}
\hline \hline & \multicolumn{4}{c}{ Open Circuit Test (OC) } \\
& \multicolumn{2}{c}{ Without shield } & \multicolumn{2}{c}{ With shield } \\
\hline Frequency & $\mathbf{L}_{\mathbf{m}}(\mu \mathbf{H})$ & $\mathbf{R}_{\mathbf{c}}(\mathbf{k} \boldsymbol{\Omega})$ & $\mathbf{L}_{\mathbf{m}}(\boldsymbol{\mu} \mathbf{H})$ & $\mathbf{R}_{\mathbf{c}}(\mathbf{k} \boldsymbol{\Omega})$ \\
$100 \mathrm{kHz}$ & 677.5 & 11.6 & 677.2 & 11.9 \\
$200 \mathrm{kHz}$ & 720.8 & 10.9 & 720.06 & 11.02 \\
$300 \mathrm{kHz}$ & 807.7 & 9.24 & 805.5 & 9.34 \\
\hline \hline
\end{tabular}

TABLE II

ShORT Circuit TESTING RESUlts OF THE COAXIAL TRANSFORMER

\begin{tabular}{|c|c|c|c|c|}
\hline & \multicolumn{4}{|c|}{ Short Circuit Test (SC) } \\
\hline & \multicolumn{2}{|c|}{ Without shield } & \multicolumn{2}{|c|}{ With shield } \\
\hline Frequency & $\mathbf{L}_{\mathbf{s}}(\mu \mathbf{H})$ & $\mathbf{R}_{\mathrm{s}}(\mathbf{\Omega})$ & $\mathbf{L}_{\mathbf{s}}(\mu \mathbf{H})$ & $\mathbf{R}_{\mathrm{s}}(\mathbf{\Omega})$ \\
\hline $100 \mathrm{kHz}$ & 1.837 & 0.111 & 1.929 & 0.119 \\
\hline $200 \mathrm{kHz}$ & 1.9 & 0.131 & 1.983 & 0.141 \\
\hline $300 \mathrm{kHz}$ & 1.88 & 0.16 & 1.976 & 0.173 \\
\hline
\end{tabular}

\section{Simulation Results AND VALIDATION}

\section{A. Eddy Current Distribution}

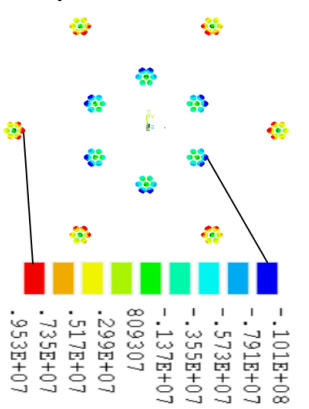

(a) Without shield

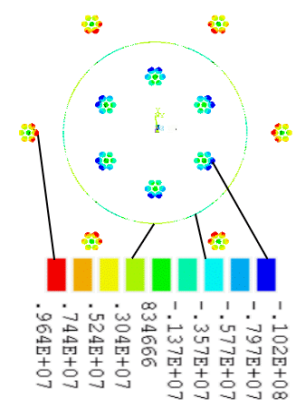

(b) With shield
Fig.4. 2D Eddy current distribution - short circuit condition. 
The 2D magnetic flux distribution of the HFCT has been presented in another paper [11]. The insertion of the Faraday shield does not affect the magnetic performance under both short circuit and open circuit conditions. This is because the magnetic field around the winding is almost perpendicular to the normal direction of shield conductor surface or parallel to the thin copper shield. As well as the thin copper shield which minimize the side effect of the shield insertion. The eddy current simulation result $(100 \mathrm{kHz}$, in frequency domain) is shown in this paper by using the outer circular winding as the primary winding and the inner circular winding as the secondary winding. Results in this paper only show the HFCT under short circuit conditions, as the impact of the Faraday shield insertion is more obvious than the HFCT under open circuit condition. A 2D simulation (see Fig. 4) shows that the peak current only increases $1.15 \%$ due to the insertion of the Faraday shield. This is because the inserted shield counteracts the proximity effect.

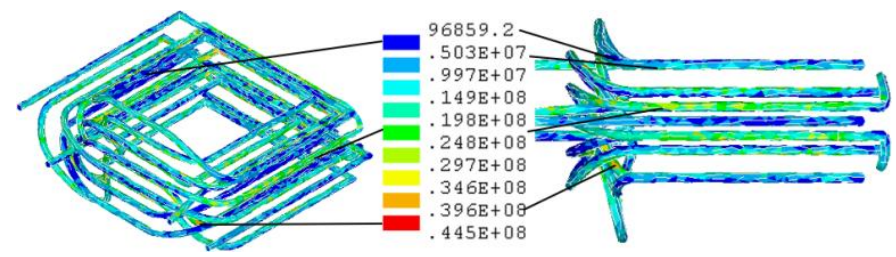

(a) Bird view

(b) Side view

Fig.5. 3D Eddy current distribution without the Faraday shield - short circuit condition.

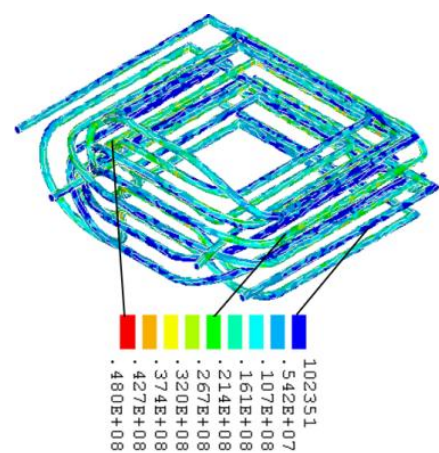

(a) Windings

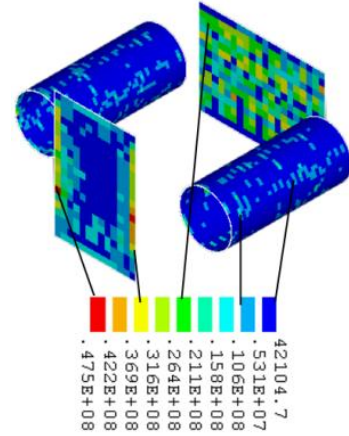

(b) Faraday shield
Fig.6. 3D Eddy current distribution with the Faraday shield - short circuit condition.

The simulation results of the 3D model, with and without the Faraday shield, are shown in Fig. 5 and 6 respectively. The peak current density increases $7.87 \%$ due to the inserted shield. The difference between the 2D and 3D simulation is mainly caused by the wire specification. A 7 strands Litz wire was used for the 2D simulation, and corresponds to the practical prototype while the 3D simulation uses the single solid conductor windings. The simplification of 3D model is to avoid the redundant computation time and complex meshing processes (it takes about 2 weeks for one HFCT simulation with Litz wires, and the meshing process requires a lot of memories). Therefore, in order to depict the electric behavior of the transformer efficiently, a 2D simulation should always be conducted before doing the full model simulation.

\section{B. Capacitance Calculation}

In order to reduce the coupling effect accompanied by the increased operating frequency, a Faraday shield was inserted. The capacitance simulation result is shown in Table III, where $\mathrm{C}_{\mathrm{ps}}$ denotes the total intra-winding capacitance, $\mathrm{C}_{\mathrm{pg}}$ and $\mathrm{C}_{\mathrm{gs}}$ represent the capacitance of the primary and secondary winding to the Faraday shield, and $\mathrm{C}_{\mathrm{ps}}$ ' denotes the intra-winding capacitance of the HFCT with the Faraday shield inserted (refer to Fig. 3 for a capacitance definition). The inserted shield must be perfectly connected to the ground, therefore the HF noise flows in the individual loop instead of propagating to the other. The $\mathrm{C}_{\mathrm{ps}}$ ' of the HFCT with the Faraday shield under grounding conditions cannot be directly measured. Therefore, $\mathrm{C}_{\mathrm{pg}}, \mathrm{C}_{\mathrm{gs}}$ and $\mathrm{C}_{\mathrm{ps}}$ need to be measured first, and $\mathrm{C}_{\mathrm{ps}}$, can then be derived based on the simple theoretical capacitance equation.

A HAMEG HM8118 LCR meter ( $20 \mathrm{~Hz}$ to $200 \mathrm{kHz})$ was used to obtain the transformer capacitance at low frequency. This is to ensure that the simulated and tested results are comparable as the simulation was done using a static field analysis (a charging equation was deployed for the solution). The capacitance decreases while the operating frequency increases. Therefore, if a static test is conducted, the testing result will be closer to the simulation result.

TABLE III CAPACITANCE OF THE HFCT

\begin{tabular}{lcllll}
\hline \hline \multicolumn{5}{c}{ Capacitance $(p \mathbf{F})$} & With Shield \\
& $\begin{array}{c}\text { Without } \\
\text { Shield }\end{array}$ & \multicolumn{4}{c}{} \\
\hline & $\mathbf{C}_{\mathbf{p s}}$ & $\mathbf{C}_{\mathbf{p g}}$ & $\mathbf{C}_{\mathbf{g s}}$ & $\mathbf{\mathbf { C } _ { \mathbf { p s } }}$ & $\mathbf{C}_{\mathbf{p s}}$ \\
2D-Coaxial & 20.4 & 41.8 & 42.3 & $1.06 \mathrm{E}-12$ & 21.02 \\
2D-End Region & 47.2 & 144.35 & 144.35 & 0.715 & 72.175 \\
2D-Sum & 67.6 & 186.15 & 186.65 & 0.715 & 93.195 \\
3D-Sum & 54.64 & 104.16 & 87.27 & 7.93 & 55.41 \\
Testing-20 Hz & 48.9 & 99.70 & 92.30 & 9.49 & 57.42 \\
\hline \hline
\end{tabular}

In Table III, simulation results are apparently higher than the testing result. This is because of the composition of the dielectric material, which exists between windings. In the practical prototype, the dielectric material (fiberglass has been used for the introduced HFCT) has a lot of tiny air gaps, which the simulated model does not have. In addition, the 2D simulation result is higher than the $3 \mathrm{D}$ result due to the length of the end region section. In a 2D simulation, the wire lengths are equal since only the $\mathrm{X}$ and $\mathrm{Y}$ axe have been taken into account (the wiring at the turning corner and in the end region section was assumed to be no difference, which is not simulated the practice).

Furthermore, capacitance is greater in the end region section than in the coaxial section due to the wiring distance being smaller in the end region section. All results again proves that it might cause a more serious EMI problem $\left(\mathrm{C}_{\mathrm{ps}}\right.$ is greater than the HFCT without the shield) if the HFCT with the Faraday shield is not carefully grounded. Lastly, testing and the 3D simulation show that the intra-winding capacitance has been greatly reduced $80.6 \%$ and $85.5 \%$ respectively.

\section{Inductance Calculation}

The benefit of computer simulation is that it not only simulates the electric and magnetic behavior of the HFCT without building real prototypes, but it also gives more detailed information, which the traditional testing method is not able to 
provide. Table IV shows the inductance result of the HFCT in $2 \mathrm{D}$ and $3 \mathrm{D}$ simulations, as well as the practical test. $\mathrm{L}_{\mathrm{m}}$ is almost identical for both testing results and simulations. The slight difference between the testing result and the simulations is caused by the precision of the simulated model and the permeability definition of the magnetic core material. The difference in the inductance between the 2D and 3D simulated results varies from $6 \%$ to $21 \%$. This difference is mainly caused by the corner connection between the coaxial section and the end region section. The simulation result in $2 \mathrm{D}$ assumes all conductors are in a straight array and thus the 3D simulations are more consistent with reality. Furthermore, a more precise simulation model can be used to reduce the difference between testing results and simulations.

TABLE IV

INDUCTANCE OF THE HFCT

\begin{tabular}{lllll}
\hline \hline & \multicolumn{2}{c}{ Wnductance $(\boldsymbol{u} \mathbf{H})$} & \multicolumn{2}{c}{ With Shield } \\
& $\mathbf{L}_{\mathbf{m}}(\boldsymbol{u} \mathbf{H})$ & $\mathbf{L}_{\mathbf{s}}(\boldsymbol{u} \mathbf{H})$ & $\mathbf{L}_{\mathbf{m}}(\boldsymbol{u} \mathbf{H})$ & $\mathbf{L}_{\mathbf{s}}(\boldsymbol{u} \mathbf{H})$ \\
\hline 2D-Coaxial & 680.75 & 0.4209 & 680.7492 & 0.409313 \\
2D-End Region & $\mathrm{N} / \mathrm{A}$ & 0.7905 & $\mathrm{~N} / \mathrm{A}$ & 0.59714 \\
2D-Sum & 680.75 & 1.2114 & 680.7492 & 1.0065 \\
3D-Sum & 678.872 & 1.28875 & 679.598 & 1.2874 \\
Testing-100 kHz & 677.5 & 1.813 & 677.2 & 1.929 \\
\hline \hline
\end{tabular}

\section{DISCUSSION}

The power losses comparison of the HFCT with and without the Faraday shield is tabulated in Table V. The power losses increase by $13.16 \%$ (short circuit) and decreases by $11.17 \%$ (open circuit) respectively for the HFCT with and without the Faraday shield. The majority of power losses occur under short circuit conditions, which are commonly called copper losses. The copper loss of the Faraday shield is $1.03 \mathrm{~W}$ under short circuit conditions and winding losses have increased by $3.48 \%$ due to the inserted shield. The increased losses are too small to be significant compared to the overall power rating of the HFCT $(8 \mathrm{~kW})$. Furthermore, the Faraday shield has a positive impact on the electric field of the HFCT under open circuit conditions. The peak eddy current decreases while the HFCT with the shield is at open circuit condition. Thus, the power loss is consequently lower than the HFCT without the Faraday shield under open circuit conditions.

TABLE V

POWER LOSSES OF THE HFCT - 3D SIMULATION

\begin{tabular}{lllll}
\hline \hline & \multicolumn{4}{c}{ Power Loss (Watt) } \\
& Without & Shield & With Shield & \\
& SC & OC & SC & OC \\
\hline Inner Winding & 5.658 & 3.257 & 5.764 & 2.644 \\
Outer Winding & 5.117 & 1.622 & 5.399 & 1.686 \\
Top Shield & N/A & N/A & 0.273 & $2.07 \mathrm{E}-3$ \\
Bottom Shield & N/A & N/A & 0.576 & $1.53 \mathrm{E}-3$ \\
Left Shield & N/A & N/A & 0.093 & $7.50 \mathrm{E}-5$ \\
Right Shield & N/A & N/A & 0.088 & $1.63 \mathrm{E}-5$ \\
Total & 10.775 & 4.879 & 12.193 & 4.334 \\
\hline \hline
\end{tabular}

The magnetic loss of the ferrite ring is also obtainable via simulations with the calculated maximum flux density. The maximum flux density has no difference in both the HFCT with or without the shield. Therefore, magnetic flux simulations are not presented in this paper. In the observation of both simulations and testing results, the Faraday shield has little impact on power losses, eddy current distribution and inductance of the HFCT. The increased power loss due to the inserted Faraday shield can be reduced by having a thinner copper shield. However, by taking the manufacturing difficulty and costs into account, as well as shield thickness limitations, the appropriate thickness can be determined with further simulation works.

Based on both the simulations and testing results, the target of reducing the $\mathrm{C}_{\mathrm{ps}}$ has been achieved. The $\mathrm{C}_{\mathrm{ps}}$ reduces from $48.9 \mathrm{pF}$ to $9.49 \mathrm{pF}$, which results in an $80.6 \%$ reduction in intra-winding capacitance. In the existing prototype, only a single layer of the Faraday shield was inserted. The number of layers should be increased if the $\mathrm{C}_{\mathrm{ps}}$ requires further reduction. However, the increased numbers from the Faraday shield also cause a reduction in HFCT efficiency.

In summary, this paper introduced a HFCT, which is suitable to replace the existing bulky and inefficient transformer in the power converter of the EV. In addition, the analysis presented in this paper might be of significant interest to other researchers as it validates the application from both electric and magnetic fields. The contribution of our research work not only brings a real prototype to the automobile industry, but also shows a pathway on power applications analysis for research studies.

\section{ACKNOWLEDGMENT}

The success of the presented work was due to the support provided by Associate Professor Steven O'Keefe. We would like to also thank my research colleague Dr. Sascha Stegen for his advice and assistance and the Head of the research centre at Griffith University, Professor David Thiel for his support in attending the 12th MMM/INTERMAG conference at Chicago.

\section{REFERENCES}

[1] C. C. Chan, "The State of the Art of Electric, Hybrid, and Fuel Cell Vehicles," PROCEEDINGS OF THE IEEE, Vol. 95, No. 4, April. 2007.

[2] F. Marra, D. Sacchetti, C. Traeholt and E. Larsen, "Electric Vehicle Requirements for Operation in Smart Grids," Innovative Smart Grid Technologies (ISGT Europe), Dec. 2011.

[3] R. Falk and S. Fries, "Electric Vehicle Charging Infrastructure - Security Considerations and Approaches," INTERNET 2012, The Fourth International Conference on Evolving Internet, pp. 58-64, June. 2012.

[4] K. Morrow, D. Karner and James Francfort, "U.S. Department of Energy Vehicle Technologies Program - Advanced Vehicle Testing Activity. Plug-in Hybrid Electric Vehicle Charging Infrastructure Review," Tech. Review, The Idaho National Laboratory, INL/EXT-08-15058, Nov. 2008.

[5] R. Torrico-Bascopé, G. Torrico-Bascopé, C. Branco, D. Ferreira and F. de Souza, "Analysis and Proposal of an Isolated DC/AC System using Three-State Switching Cell," IEEE Power Electronics Conference, pp. 536-543, 2009.

[6] H. Jou, W. Chiang and J. Wu, "Voltage-Mode Grid-Connected Solar Inverter with High Frequency Isolated Transformer," IEEE International Symposium on Industrial Electronics (ISIE 2009), pp. 1087-1092, July. 2009.

[7] W. Merwe and T. Mouton, "Solid-State Transformer Topology Selection," IEEE International Conference on Industrial Technology (ICIT 2009), pp. 1-6, Feb. 2009.

[8] L.Dalessandro, F. D. S.Cavalcante, and J. W. Kolar, "Self-Capacitance of High-Voltage Transformers", IEEE Trans.on PowerElectronics, Vol. 22, No. 5, pp. 2081-2092, Sept. 2007.

[9] J. Lu and F. Dawson, "Analysis of Eddy Current Distribution in High Frequency Coaxial Transformer with Faraday Shield," IEEE Trans. On Magnetics, Vol. 42, No. 10, pp. 3171-3173. Oct. 2006

[10] J. Lu, "High Frequency and High Power Density Magnetic Devices and Future Trends", International Conference on Power Electronics and Motion (PCIM2009), invited paper, April. 2009.

[11] S. Stegen and J. Lu, "Shielding Effect of High Frequency Power Transformers for DC/DC Converters used in Solar PV Systems," Proceedings of IEEE APEMC2010, pp. 414-417, April, 2010. 